For Publisher's use

\title{
SEARCHES FOR SUPERSYMMETRY AT THE TEVATRON
}

\author{
ARND MEYER \\ III. Phys. Inst. A, RWTH Aachen, 52074 Aachen, Germany \\ E-mail: meyera@fnal.gov \\ (for the DØ and CDF Collaborations)
}

\begin{abstract}
Both Tevatron experiments, $\mathrm{D} \varnothing$ and CDF, have searched for signs of Supersymmetry in the present Run II data sample, using integrated luminosities of up to $260 \mathrm{pb}^{-1}$ collected in $p \bar{p}$ collisions at a centerof-mass energy of $1.96 \mathrm{TeV}$. In these proceedings, new results are presented in the search for squarks and gluinos in the jets and missing transverse energy final state, associated production of charginos and neutralinos with multilepton final states, search for the rare decay $B_{s} \rightarrow \mu \mu$, searches allowing R-parity violation (muons+jets, multileptons), and searches in the gauge mediated supersymmetry breaking framework with the final state of two photons and missing transverse energy. In the absence of any significant deviation from Standard Model expectations, limits on the presence of new physics are set, which in many cases are the most stringent to date.
\end{abstract}

After the very successful Tevatron Run I, the accelerator and the colliding beam experiments CDF and D $\varnothing$ were upgraded for Run II. On the accelerator side, the beam energy has been increased from $900 \mathrm{GeV}$ to $980 \mathrm{GeV}$. A new proton storage ring, the "Main Injector", has been built, and the same tunnel houses the "Recycler" storage ring, which will improve the rate at which antiprotons can be accumulated. The bunch spacing in the Tevatron has been reduced from $3.5 \mu$ s to $396 \mathrm{~ns}$. The results shown below ${ }^{1}$ are based on early Run II data collected in 2002-2004 and correspond to an integrated luminosity of up to $260 \mathrm{pb}^{-1}$, significantly larger than the Run I data $\operatorname{set}\left(\int \mathcal{L} d t \simeq 110 \mathrm{pb}^{-1}\right)$.

\section{Search for New Physics with Jets $+\boldsymbol{E}_{T}$}

Both CDF and $\mathrm{D} \varnothing$ have reported preliminary results in signatures with jets and $\mathbb{E}_{T}$. In the light quark jets $+\mathbb{E}_{T}$ search based on $85 \mathrm{pb}^{-1}$ of $\mathrm{D} \varnothing$ data, two high $E_{T}$ jets and large $H_{T}>275 \mathrm{GeV}$ are required, and $\mathbb{E}_{T}$ $>175 \mathrm{GeV}$ (Fig. 1). It is worth noting the single event in the tail of the $\mathbb{E}_{T}$ distribution: it has four jets with $E_{T}$ of $289 \mathrm{GeV}, 117 \mathrm{GeV}$, $14 \mathrm{GeV}$, and $11 \mathrm{GeV}$, and $\mathbb{E}_{T}=381 \mathrm{GeV}$. After all cuts, 4 events remain in the data, with

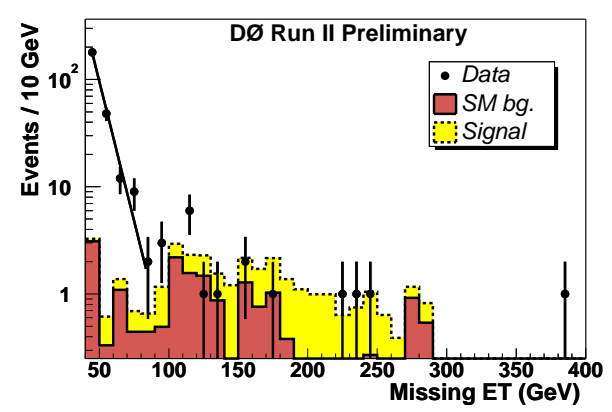

Figure 1. Distribution of $E_{T}$ in the $\mathrm{D} \varnothing$ acoplanar jets $+E_{T}$ analysis to search for squarks and gluinos.

$2.67 \pm 0.95$ expected from Standard Model processes. The dominant background is due to $Z \rightarrow \nu \nu+2$ jets. Limits are set on the production of light squarks and gluinos in an mSUGRA scenario $\left(m_{0}=25 \mathrm{GeV}, A_{0}=0\right.$, $\tan \beta=3, \mu<0): 292 \mathrm{GeV}$ for squarks, and $333 \mathrm{GeV}$ for gluinos, beyond the Run I excluded domain.

CDF has searched for pair production of gluinos and decays gluino $\rightarrow$ sbottom + bottom in $156 \mathrm{pb}^{-1}$ of data. The analysis requires four jets and large $\mathbb{E}_{T}$, and one or two b-tags. In the more sensitive double tag analysis, 4 events are observed with $\mathbb{E}_{T}>80 \mathrm{GeV}$, where $2.6 \pm 0.7$ events are expected from Standard Model processes (mostly top quark pair production). The exclusion limits, signifi- 


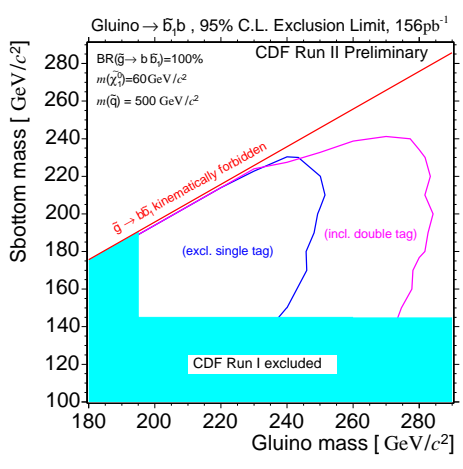

Figure 2. The 95\% C.L. exclusion region from the CDF four jet $+E_{T}$ search for gluino pair production and decay into sbottom squarks.

cantly extending earlier results, in the gluino sbottom mass plane are shown in Fig. 2.

\section{Search for $B_{s}^{0} \rightarrow \mu^{+} \mu^{-}$}

The purely leptonic decays $B_{d, s}^{0} \rightarrow \mu^{+} \mu^{-}$are flavor-changing neutral current processes. In the Standard Model, these decays are forbidden at the tree level and proceed at a very low rate through higher-order diagrams. However, the decay amplitude can be enhanced in some extensions of the Standard Model. In the MSSM for example $\mathcal{B}\left(B_{s}^{0} \rightarrow \mu^{+} \mu^{-}\right) \propto$ $(\tan \beta)^{6}$, leading to an enhancement of up to three orders of magnitude.

Both DØ and CDF have reported new results of a search for the decay $B_{s}^{0} \rightarrow \mu^{+} \mu^{-}$. Using a data set with integrated luminosity of $240 \mathrm{pb}^{-1}, \mathrm{D} \varnothing$ sets an upper limit on the branching fraction of $\mathcal{B}\left(B_{s}^{0} \rightarrow \mu^{+} \mu^{-}\right) \leq$ $5.0 \times 10^{-7}$ at the $95 \%$ C.L., the most stringent upper bound to date. Based on $171 \mathrm{pb}^{-1}$ of data, CDF determined $\mathcal{B}\left(B_{s}^{0} \rightarrow \mu^{+} \mu^{-}\right)<$ $7.5 \times 10^{-7}$ at the $95 \%$ C.L. ${ }^{2}$.

\section{Associated Production of Charginos and Neutralinos}

The three-lepton signature $\left(+\mathbb{E}_{T}\right)$ is a gold plated signature for the associated production of charginos and neutralinos. D $\varnothing$ has presented four different analyses, in the end

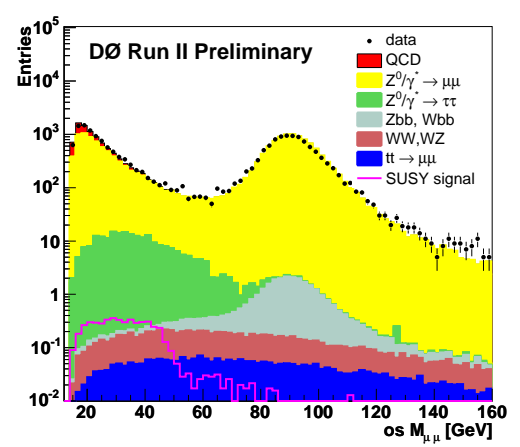

Figure 3. Invariant dimuon mass spectrum in the $\mathrm{D} \varnothing$ search for associated production of charginos and neutralinos.

combined for maximum sensitivity. The four analyses are based on the signature of two electrons plus an isolated track, two muons plus an isolated track, one electron plus one muon plus an isolated track, and finally two muons with the same charge. The isolated track requirement is sensitive to electrons, muons, and taus, and maximizes efficiency by not requiring explicit lepton identification. All identified leptons are required to be isolated. The invariant dimuon mass spectrum is shown in Fig. 3, illustrating background and expected signal contributions. Challenges for the analyses are the very small signal cross sections $(\sigma \times \mathrm{BR}<0.5 \mathrm{pb})$ and typically low lepton momenta due to cascade decays. The data samples used correspond to $147-249 \mathrm{pb}^{-1}$. After all cuts, 1, 1, 0, and 1 event remain in the data, compatible with the Standard Model expectations of $0.7 \pm 0.5$, $1.8 \pm 0.5,0.3 \pm 0.3$, and $0.1 \pm 0.1$ events.

The derived cross section limits, shown in Fig. 4, are significantly more stringent than Run I results. The corresponding chargino mass limit is close to the LEP limits, for an mSUGRA scenario with maximum leptonic branching fractions .

\section{R-parity Violation}

Supersymmetry does not impose the conservation of lepton and baryon numbers. Al- 
For Publisher's use

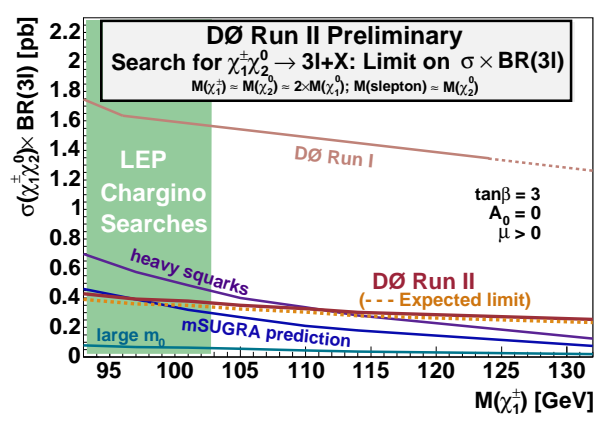

Figure 4. Combined $\mathrm{D} \varnothing$ limits from the search for associated production of charginos and neutralinos.

lowing R-parity to be violated leads to signatures distinct from R-parity conserving models. D $\varnothing$ has performed searches for not vanishing $L L \bar{E}$ couplings $\lambda_{121}, \lambda_{122}$ and $L Q \bar{D}$ coupling $\lambda_{211}^{\prime}$. For non-zero $L L \bar{E}$ coupling SUSY particles are produced in pairs or associated, and (cascade) decay to the lightest neutralino $\tilde{\chi}_{0}^{1}$, which then decays into two charged leptons and one neutrino by violating R-parity. The final state includes four charged leptons (electrons or muons for $\lambda_{121}$ and $\lambda_{122}$ ) and two neutrinos which lead to missing transverse energy.

The $L Q \bar{D}$ coupling offers the opportunity to produce the scalar supersymmetric particles as resonances. For $\lambda_{211}^{\prime}$ this is either a smuon or a muon sneutrino. The smuon can decay into the lightest neutralino $\tilde{\chi}_{0}^{1}$ and a $\operatorname{high} p_{T}$ muon. The neutralino decays via the same R-parity violating coupling $\lambda_{211}^{\prime}$ into one muon and two jets.

\subsection{R-parity Violation via $\lambda_{121}$ and $\lambda_{122}$ (eel and $\mu \mu l$ Final States)}

The data samples correspond to an integrated luminosity of $238 \mathrm{pb}^{-1}\left(\lambda_{121}\right)$ and $160 \mathrm{pb}^{-1}\left(\lambda_{122}\right)$. For the $\lambda_{121}$ analysis, the identification of two electrons and a third lepton, an electron or a muon, is required. A fourth lepton is not required. The $\mathrm{Z}$ resonance is cut away and significant $E_{T}$ is required. After all cuts no data events remain while $0.45 \pm 0.43$ events are expected from Standard Model processes.

For the $\lambda_{122}$ analysis, events with at least two muons plus an electron or a muon are selected. No fourth lepton is required for maximum efficiency, similar to the electron channel. Two-dimensional cuts in the invariant dimuon mass, $\mathbb{E}_{T}$, and the sum of muon $p_{T}$ are applied to improve the sensitivity. Two events are found in the data, while $0.63 \pm 1.93$ events are expected from Standard Model processes.

\subsection{R-parity Violation via $\lambda_{211}^{\prime}$ ( $\mu \mu$ jet jet)}

The search in the resonant channel uses data with a luminosity of $154 \mathrm{pb}^{-1}$. The data sample contains events with two well measured muons and two jets. The slepton and neutralino masses are reconstructed, since all decay products are detected. The neutralino mass is calculated using the two jets and the next to leading muon, since the muon created at the slepton decay vertex together with the neutralino is with high propability the leading $p_{T}$ muon. Sliding cuts on the leading muon transverse momentum and the reconstructed $\mathrm{Z}, \tilde{\chi}_{0}^{1}$ and $\tilde{l}$ masses are applied depending on the neutralino and slepton mass of the SUSY parameter point under study. No excess above the Standard Model expectation is observed.

\subsection{Results}

In the absence of an excess in the data, upper limits are set on the production cross sections. The cross section limits are translated into limits on the gaugino and slepton masses (see examples in Table 1 and Fig. 5).

\section{Gauge Mediated SUSY Breaking}

In these analyses, perfomed both by CDF and $\mathrm{D} \varnothing$ with $202 \mathrm{pb}^{-1}$ and $263 \mathrm{pb}^{-1}$, respectively, the NLSP is assumed to be the lightest neutralino. The decay of the neutralinos into 


\begin{aligned}$\lambda_{121}=0.01 & \tan \beta=5 \\$\hline$\mu<0: & M_{\tilde{\chi}^{ \pm}}<184 \mathrm{GeV} \\ \mu>0: & M_{\tilde{\chi}^{ \pm}}<181 \mathrm{GeV} \\$\hline \hline$\lambda_{122}=0.001 & \tan \beta=5 \\$\hline$\mu<0: & M_{\tilde{\chi}^{ \pm}}<160 \mathrm{GeV} \\ \mu>0: & M_{\tilde{\chi}^{ \pm}}<165 \mathrm{GeV} \\$\hline \hline$\lambda_{211}^{\prime}=0.07 & \tan \beta=2, \mu<0 \\$\hline$M_{\tilde{l}}=200 \mathrm{GeV}: & 54<M_{\tilde{\chi}_{1}^{0}}<110 \mathrm{GeV} \\ M_{\tilde{\chi}_{0}^{1}}=75 \mathrm{GeV}: & 160<M_{\tilde{l}}<255 \mathrm{GeV} \\ M_{\tilde{\chi}_{0}^{1}}=100 \mathrm{GeV}: & 190<M_{\tilde{l}}<255 \mathrm{GeV}\end{aligned}$

Table 1. D $\varnothing$ results on excluded gaugino and slepton masses with $95 \%$ C.L. for the R-parity violating coupling parameters $\lambda_{121}, \lambda_{122}$ and $\lambda_{211}^{\prime}$.

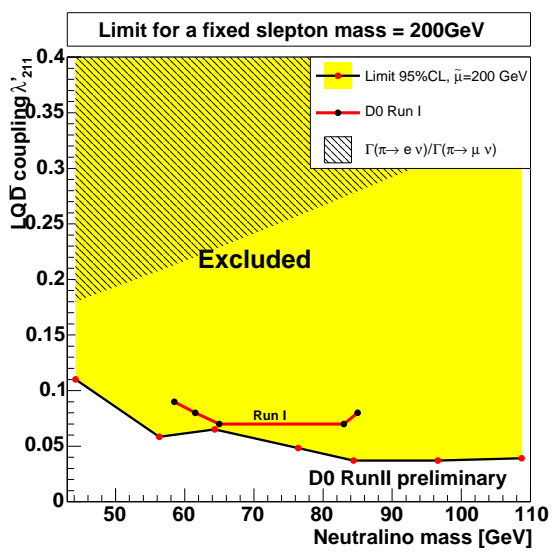

Figure 5. D $\varnothing$ search for non-vanishing $\lambda_{211}^{\prime}$ coupling, $95 \%$ C.L. limits on the lightest neutralino mass for a fixed $M_{\tilde{l}}=200 \mathrm{GeV}$.

gravitino and photon leads to a final state with two photons and $\mathbb{E}_{T}$. The dominant backgrounds are from direct photon production and from jets misidentified as a photon (Fig. 6). CDF (DØ) requires two photons with $E_{T}>13(20) \mathrm{GeV}$ in the central part of the detector, and $\mathbb{E}_{T}>45(40) \mathrm{GeV}$. The experiments observe $0(\mathrm{CDF})$ and $2(\mathrm{D} \varnothing)$ events in the data, with $0.6(\mathrm{CDF})$ and 3.7 $(\mathrm{D} \varnothing)$ events expected in the Standard Model. For the number of messengers $N=1$, the messenger mass $M_{m}=2 \Lambda, \mu>0$, and $\tan \beta=15$, the limits on the neutralino mass (Fig. 7) are $93 \mathrm{GeV}(\mathrm{CDF})$ and $108 \mathrm{GeV}(\mathrm{D} \varnothing)$,

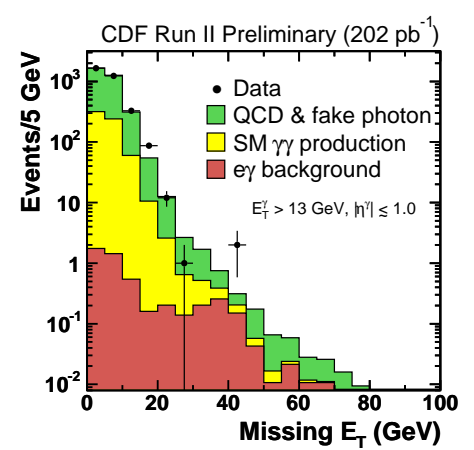

Figure 6. The $E_{T}$ distribution for the CDF $\gamma \gamma$ data sample.

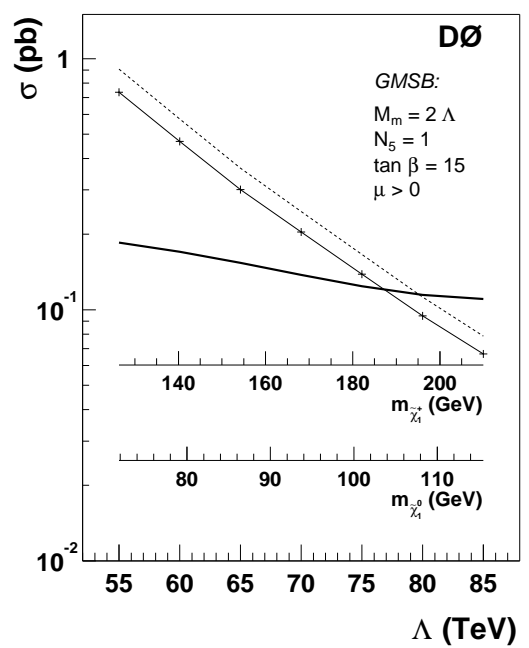

Figure 7. The $95 \%$ C.L. exclusion region from D $\varnothing$ in the $\gamma \gamma+E_{T}$ search for GMSB SUSY.

extending beyond the LEPII limit.

\section{References}

1. Details for all results can be found at http://www-d0.fnal.gov/Run2Physics/ WWW/results/NP/np.htm, http://www-cdf.fnal.gov/physics/ exotic/exotic.html

2. CDF Collaboration, Phys. Rev. Lett. 93, 032001 (2004)

3. DØ Collaboration, Search for Supersymmetry with Gauge-Mediated Breaking in Diphoton Events at D $\varnothing$, submitted to Phys. Rev. Lett. 